\title{
Differential expression of PD-L1 and IDO1 in association with the immune microenvironment in resected lung adenocarcinomas
}

\author{
M. Lisa Zhang $\mathbb{1}^{1} \cdot$ Marina Kem ${ }^{1} \cdot$ Meghan J. Mooradian ${ }^{2,3} \cdot$ Jean-Pierre Eliane ${ }^{1} \cdot$ Tiffany G. Huynh ${ }^{1}$. \\ A. John lafrate ${ }^{1,2,4} \cdot$ Justin F. Gainor ${ }^{2,3} \cdot$ Mari Mino-Kenudson $\mathbb{D}^{1,2,4}$
}

Received: 11 June 2018 / Revised: 18 September 2018 / Accepted: 19 September 2018 / Published online: 26 October 2018

(c) United States \& Canadian Academy of Pathology 2018

\begin{abstract}
Like programmed cell death ligand 1 (PD-L1), indoleamine 2,3-dioxygenase 1 (IDO1) is known to exert immunosuppressive effects and be variably expressed in human lung cancer. However, IDO1 expression has not been well studied in lung adenocarcinoma. PD-L1 and IDO1 expression was evaluated in 261 resected lung adenocarcinomas using tissue microarrays and H-scores (cutoff: 5). We compared IDO1 and PD-L1 expression with clinical features, tumor-infiltrating lymphocytes, HLA class I molecule expression, molecular alterations, and patient outcomes. There was expression of PD-L1 in 89 (34\%) and IDO1 in 74 (29\%) cases, with co-expression in $49(19 \%)$. Both PD-L1 and IDO1 were significantly associated with smoking, aggressive pathologic features, and abundant CD8+ and T-bet + (Th1 marker) tumor-infiltrating lymphocytes. PDL1 expression was also associated with preserved HLA class I molecule expression $(p=0.002)$. Compared to PD-L1 $+/$ IDO1 + and PD-L1+ only cases, significantly fewer IDO1+ only cases had abundant CD8 + and T-bet + tumorinfiltrating lymphocytes $(p<0.001$, respectively). PD-L1 expression was significantly associated with $E G F R$ wild-type $(p<$ $0.001)$ and KRAS mutants $(p=0.021)$, whereas isolated IDO1 expression was significantly associated with EGFR mutations $(p=0.007)$. As for survival, PD-L1 was a significant predictor of decreased progression-free and overall survival by univariate but not multivariate analysis, while IDO1 was not associated with progression-free or overall survival. Interestingly, there was a significant difference in the 5-year progression-free and overall survival $(p=0.004$ and 0.038 , respectively), where cases without PD-L1 or IDO1 expression had the longest survival, and those with PD-L1 alone had the shortest survival. While PD-L1+/-IDO1 expression is observed in association with HLA class I expression, cytotoxic T lymphocyte/Th1 microenvironments, EGFR wild-type, and KRAS mutations, isolated IDO1 expression does not demonstrate these associations, suggesting that IDO1 may serve a distinct immunosuppressive role in lung adenocarcinomas. Thus, further investigation of IDO1 may demonstrate its role as a potential biomarker for patients who undergo anti-PD-1/PD-L1 therapy.
\end{abstract}

Electronic supplementary material The online version of this article (https://doi.org/10.1038/s41379-018-0160-1) contains supplementary material, which is available to authorized users.

Mari Mino-Kenudson

mminokenudson@partners.org

1 Department of Pathology, Massachusetts General Hospital, Boston, MA, USA

2 Cancer Center, Massachusetts General Hospital, Boston, MA, USA

3 Department of Medicine, Harvard Medical School, Boston, MA, USA

4 Department of Pathology, Harvard Medical School, Boston, MA, USA

\section{Introduction}

Recent therapeutic strategies for non-small cell lung cancer have focused on targeting immune checkpoints to reinvigorate the host immune system and thus restore anti-tumor responses. Most notably and extensively studied across many malignancies is the programmed cell death 1 (PD-1) receptor/programmed cell death ligand 1 (PD-L1) interaction, which suppresses the CD8+ cytotoxic T-cell immune response, thus allowing tumor cells to bypass immune surveillance [1]. Multiple major clinical trials [2-6] have demonstrated significant improvements in patient survival in response to anti-PD-1 (i.e., nivolumab, pembrolizumab) and anti-PD-L1 (i.e., atezolizumab) agents. As a result, these agents are now FDA (Food and Drug Administration) 
approved for the treatment of patients with metastatic nonsmall cell lung cancer with disease progression during or after platinum-based chemotherapy. Of those, pembrolizumab has also been approved as the first-line therapy for advanced non-small cell lung cancer patients whose tumors express PD-L1 by immunohistochemistry on $50 \%$ or more of tumor cells and for advanced non-squamous nonsmall cell lung cancer patients in combination with platinum/pemetrexed regardless of PD-L1 expression [7, 8].

We have previously shown that PD-L1 expression in resected lung adenocarcinomas is associated with significant smoking history, aggressive pathologic features, the CD8+ cytotoxic $\mathrm{T}$ lymphocyte and $\mathrm{T}$ helper type 1 (Th1) immune microenvironment, and KRAS mutations and EGFR wild-type, using an individually-validated PD-L1 immunohistochemistry assay and a variety of scoring systems including H-score [9]. In that study, PD-L1 expression was predictive of decreased progression-free survival and overall survival by univariate but not multivariate analysis, though other previous studies have reported conflicting results regarding prognostication using PD-L1 expression [10-12].

Similar to PD-L1, indoleamine 2,3-dioxygenase 1 (IDO1) is known to exert a potent immunosuppressive effect and be variably expressed in a variety of human solid tumors, including non-small cell lung cancer [13, 14]. Furthermore, IDO1 has been shown to contribute to tumor progression in mouse models of lung cancer [15]. IDO1 is an enzyme with limited expression in normal adult tissues (lymphoid tissues and placenta) that catalyzes the rate-limiting step in the catabolic conversion of tryptophan to kynurenine. The depletion of tryptophan induces apoptosis/dysfunction of CD4+ T cells, and the concurrent accumulation of kynurenine promotes regulatory T-cell differentiation [16]. In vitro studies have shown that IDO1 expression is not associated with CD3+ and CD8+ T cells in a colorectal cancer cell line [17], but IDO1 inhibition in murine melanoma models lead to a marked increase in CD8+ tumor-infiltrating lymphocytes [18]. As such, tumor expression of IDO1 favors immune escape, and clinical investigation $[16,19,20]$ of IDO1 inhibitors alone or in combination with other immune-checkpoint blockade are underway in hopes of reversing tumor-induced immunosuppression via tryptophan depletion. In terms of the tumor immune microenvironment, like PD-L1, IDO1 has also been shown to increase in response to interferon- $\gamma$ (IFN $\gamma$ ) and to be associated with increased $\mathrm{CD} 3+$ and $\mathrm{CD} 8+$ tumor-infiltrating lymphocytes via quantitative immunofluorescence [21]. However, the correlation of IDO1 with additional clinicopathologic and immunologic parameters, molecular alterations, and patient outcomes in non-small cell lung cancer, as well as its relationship with PD-L1, have not been well characterized.

In this study, we compared the expression of PD-L1 and IDO1 in correlation to clinicopathologic variables, subtypes of tumor-infiltrating lymphocytes, human leukocyte antigen (HLA) class I expression, molecular alterations, and patient outcomes in a large cohort of molecularly annotated lung adenocarcinomas.

\section{Materials and methods}

\section{Study population}

The study was approved by the Massachusetts General Hospital Institutional Review Board. A total of 461 cases of primary lung adenocarcinomas resected between July 2010 and December 2012 were identified from the Massachusetts General Hospital surgical pathology database. After excluding post-neoadjuvant therapy resections, cases with concurrent multiple primaries, and those with no molecular testing or no sufficient tumor blocks available, 261 cases formed the study cohort. All cases in this cohort had undergone a multiplex polymerase chain reaction-based assay (SNaPshot platform; Applied Biosystems, Waltham, MA) to detect a panel of commonly mutated genes including EGFR, KRAS, BRAF, Her2, and TP53, as previously described [22]. Subsets of the cohort had also been tested for $A L K(n=248)$ or ROS1 $(n=65)$ gene rearrangements, or MET $(n=17), \operatorname{EGFR}(n=4)$, or Her2 $(n=2)$ amplifications. Demographic information, smoking history, and post-operative follow-up data were collected from patient records. Progression-free survival was measured from the time of resection to the development of clinical/ radiographic progression or death; patients alive without documented disease progression were censored on the date of last follow-up. Overall survival was measured from the date of resection to death due to any cause.

\section{Pathology examination and tissue microarray construction}

All tumor slides were reviewed, and tumor size, stage (pT and $\mathrm{pN}$ ), histologic pattern, nuclear grade, pleural invasion, lymphatic invasion, and vascular invasion were evaluated in each case by three pathologists who were blinded to the clinical information [9]. The histology of the tumor was classified in accordance with the International Association for the Study of Lung Cancer/American Thoracic Society/European Respiratory Society multidisciplinary classifications [23]. The tumor stage was diagnosed in accordance with the American Joint Committee on Cancer (AJCC) Cancer Staging Manual, 7th edition [24]. Nuclear grade was semi-quantitatively assessed on a scale of 1 to 3 [25].

Two-millimeter core tissue microarrays were constructed, with two cores from each of the study cases. If 
Fig. 1 Evaluation of immunohistochemical expression in lung adenocarcinomas using $\mathrm{H}$ score (cutoff $=5$ ) (magnification $\times 400$ for $\mathbf{a}-\mathbf{i}$, $\times 200$ for $\mathbf{j}-\mathbf{l})$. Membranous PD-L1: a 0 (arrowhead), 1 (arrow); b 2 (arrowhead), 3 (arrow). Cytoplasmic IDO1: c 1; d 2 (arrowhead), 3 (arrow). Membranous B2M: e 0 (arrowhead), 1 (arrow); f 2. Membranous HC10 (HLA-B/C): g 1 (arrowhead), 2 (arrow); h 2 (arrowhead), 3 (arrow). i Membranous HCA2 (HLA-A). Tumor-infiltrating lymphocytes were scored as the fraction of tumor cells with overlying positive T cells: 0 , none/rare; $1,<5 \%$ (j); $2,>5$ to $<25 \%$ (k); $3,>25 \%$ (l)

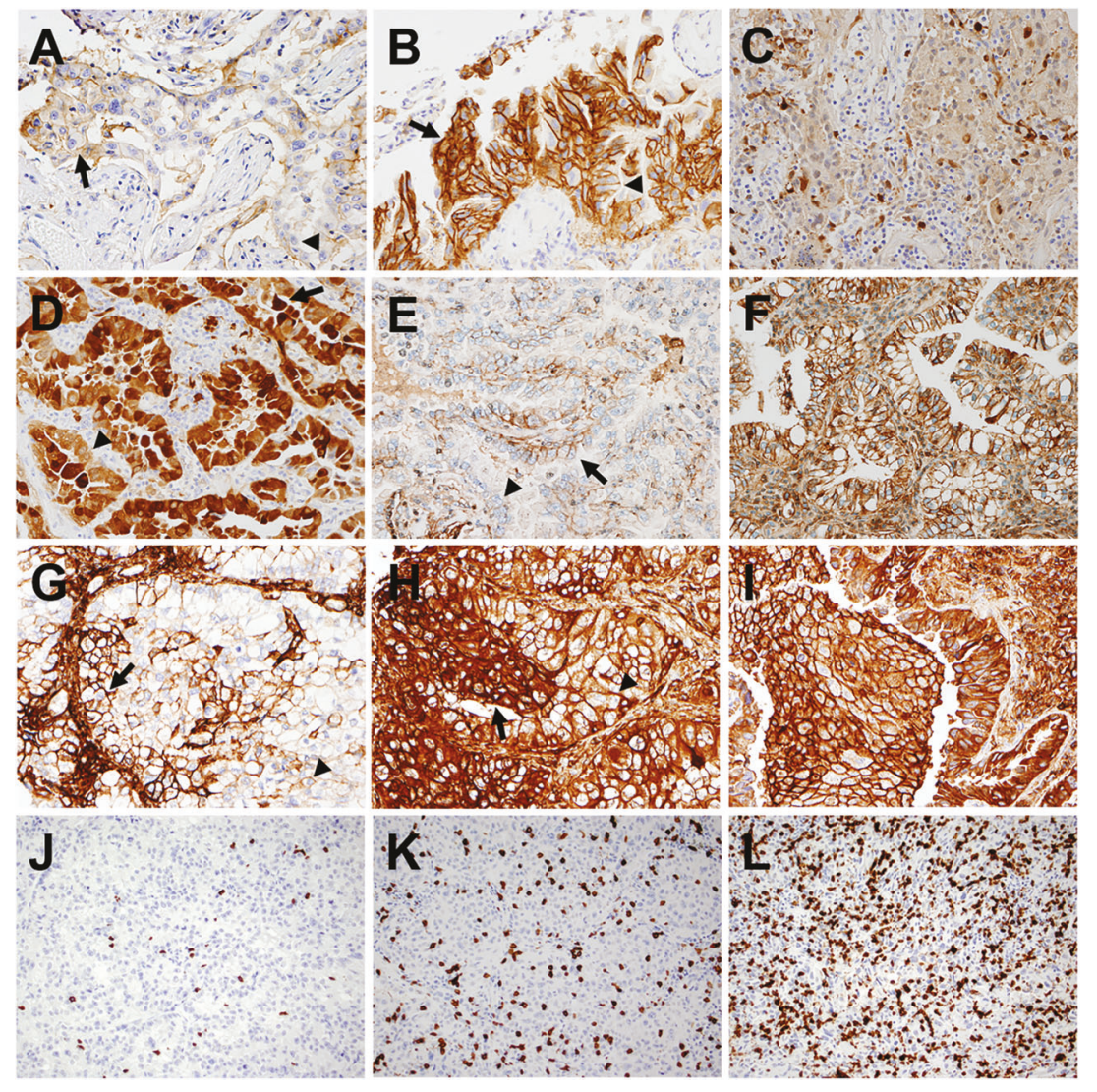

available, one core was taken from the center of the tumor and the other from the invasive front. Two cores each of tonsil and placenta (positive controls) and normal lung parenchyma were also included.

\section{Immunohistochemistry}

Immunohistochemistry was performed on $5 \mu \mathrm{m}$ sections cut from tissue microarray blocks using an automated stainer (Bond Rx; Leica Microsystems, Bannockburn, IL) and the following primary antibodies in accordance with the manufacturer's recommendations: PD-L1 (E1L3N (1:200), Cell Signaling Technology, Danvers, MA), IDO1 (1F8.2 (1:400), Millipore, Burlington, MA), B2M (D8P1H (1:8000), Cell Signaling Technology, Danvers, MA), HCA2 (IgG1 (1:100), Nordic-MUbio, The Netherlands), HC10 (IgG2a (1:100), Nordic-MUbio, The Netherlands), CD8 (4B11 (RTU), Leica Biosystems, Buffalo Grove, IL), T-bet (D6N8B (1:100), Cell Signaling Technology, Danvers, MA), and GATA3 (L50-823 (1:250), Biocare Medical, Pacheco, CA).

\section{Interpretation of expression by immunohistochemistry}

Two pathologists (MLZ and MM-K) who were blinded to the clinical and pathologic data evaluated cytoplasmic expression of IDO1 and membranous expression of B2M (the common HLA-A/B/C light chain required for surface expression), HCA2 (a specific antibody for HLA-A heavy chains), and HC10 (a specific antibody for HLA-B/C heavy chains) (Fig. 1) [26-28]. An H-score was recorded for each marker in each case. H-score was calculated based on the intensity (0-3) of membranous staining and extent (\%) of positive tumor cells $(3 \times$ percentage of strongly staining cells $+2 \times$ percentage of moderately staining cells + percentage of weakly staining cells), ranging from 0 to 300 . The expression of each immunostain was dichotomized as negative and positive based on an $\mathrm{H}$-score cutoff of 5 ( $\geq 5$ vs. <5) [29]. If discrepant scores were given to the two different cores from the same case, the final score was determined following discussion under a multi-headed microscope.

Evaluation of PD-L1 membranous staining by immunohistochemistry was independently performed by two pathologists who were blinded to the clinical and pathologic data, as previously described [9]. Similarly, two of the authors previously evaluated tumor-infiltrating lymphocytes using cytoplasmic expression of CD8 (a marker for cytotoxic $\mathrm{T}$ lymphocytes) and nuclear expression of T-bet (a marker for Th1 pathway activation) and GATA3 (a marker for Th2 pathway activation) immunohistochemistry. tumorinfiltrating lymphocytes were scored as the fraction of 
tumor cells with overlying positive T cells: 0 , none or rare; $1,<5 \% ; 2, \geq 5$ and $<25 \%$; and $3, \geq 25 \%$. Scoring of lymphocytes in the tumor stroma was performed by comparing the percentage of positive lymphocytes with the total number of nucleated cells in the stromal compartments [9].

\section{Statistical analysis}

Associations between PD-L1 and/or IDO1 expression and demographics, clinicopathologic features, tumor-infiltrating lymphocytes (CD8+, T-bet + , and GATA3+), HLA class I molecule expression, and molecular alterations were examined using Fisher's exact tests for categorical variables and the twosided $t$-test or Wilcoxon rank-sum test for continuous variables, as appropriate. Multivariate analysis was performed with a binary logistic regression model to evaluate for independent predictors of PD-L1 and IDO1 expression using an H-score cutoff of $\geq 5$ to adjust for potential confounders. Survival analyses for progression-free and overall survival were performed using the Kaplan-Meier estimator and the log-rank test. Multivariate survival analysis was performed using the Cox proportional hazards regression model to adjust for potential confounders. The $p$ values of $<0.05$ were considered statistically significant. Statistical analyses were performed using $\mathrm{R}$ version 3.2.2.

\section{Results}

\section{Correlation of PD-L1 and ID01 expression with clinicopathologic features}

The study cohort $(n=261)$ had a mean age of 68 years (standard deviation: 10), and $171(66 \%)$ were female. One case had no evaluable tumor for the assessment of IDO1 expression. Using an H-score cutoff of 5, 89/261 (34\%) and 74/260 (29\%) cases showed positive PD-L1 and IDO1 expression, respectively. Of the 260 cases, 146 (56\%) were PD-L1-/IDO1-, 40 (15\%) were PD-L1+/IDO1-, 25 (10\%) were PD-L1-/IDO1+ and 49 (19\%) were PD-L1+/IDO1+ (Fig. 2, Supplementary Table 1). The linear (Pearson) correlation coefficient between PD-L1 and IDO1 expression was $r=0.4$ (weak correlation). Clinicopathologic and molecular characteristics of the study cohort were correlated with PD-L1, IDO1, and IDO1+/-PD-L1 expression (Table 1). PD-L1 expression was significantly associated with smoking history ( $>10$ and $>30$ pack-years, $p<0.001$ for both), higher pT stage $(p=0.037)$, higher $\mathrm{pN}$ stage $(p=0.039)$, solid-predominant pattern $(p<0.001)$, higher nuclear grade $(p<0.001)$, and the presence of lymphatic invasion and venous invasion $(p<0.001$ for both). IDO1 expression was significantly associated with a history of heavy smoking $(>30$ pack-years, $p=0.018)$, acinar-predominant $(p=0.003)$ and

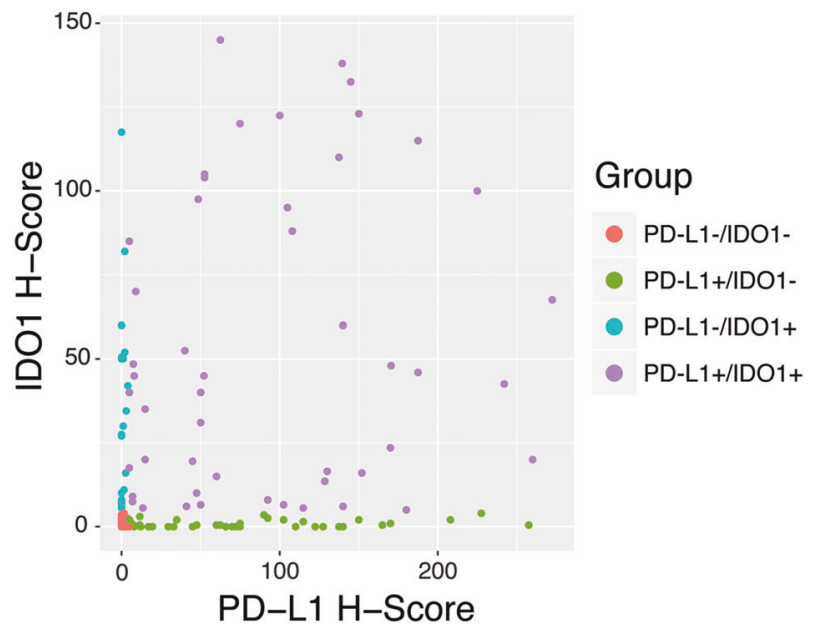

Fig. 2 Correlation between PD-L1 and IDO1 expression (cutoff of $\mathrm{H}$ score $=5$ )

solid-predominant $(p=0.042)$ patterns, higher nuclear grade $(p=0.007)$, and the presence of lymphatic invasion $(p=$ $0.011)$ and vascular invasion $(p=0.040)$. There was no association between either PD-L1 or IDO1 and the overall AJCC pathologic stage or the presence of pleural invasion.

After stratifying the IDO1+ cases by PD-L1 expression, there was no longer a significant association between isolated IDO1 expression (IDO1+/PD-L1-) and heavy smoking history, solid-predominant histologic pattern, and the presence of lymphatic or vascular invasion (Table 1). However, isolated IDO1 expression was significantly associated with acinar-predominant histologic pattern $(p<$ $0.001)$ and lower nuclear grade $(p<0.001)$. There were no differences between isolated PD-L1 expression and PD-L1/ IDO1 co-expression.

\section{Correlation of PD-L1 and ID01 expression with tumor-infiltrating lymphocytes}

As for tumor-infiltrating lymphocytes, both PD-L1 and IDO1 expression were significantly associated with abundant CD8+ tumor-infiltrating lymphocytes (66\% vs. $4 \%$ and $47 \%$ vs. $16 \%$ of cases, $p<0.001$, respectively), CD8+ lymphocytes in the tumor stroma (64\% vs. $23 \%$ and $55 \%$ vs. $29 \%$ of cases, $p<0.001$, respectively), and T-bet + tumor-infiltrating lymphocytes $(27 \%$ vs. $4 \%$ and $20 \%$ vs. $8 \%$ of cases, $p<0.001$ and $p=0.010$, respectively). There was no association of either PD-L1 or IDO1 with GATA3+ tumor-infiltrating lymphocytes, or stromal T-bet + or GATA3 + lymphocytes. Isolated PD-L1 expression (PD-L1 +/IDO-) demonstrated the same associations. Isolated IDO1 expression (IDO1+/PD-L1-) was no longer significantly associated with abundant T-bet+ tumorinfiltrating lymphocytes; all $25(100 \%)$ isolated IDO1+ cases had rare-to-few CD8+ tumor-infiltrating lymphocytes 
Table 1 Clinicopathologic characteristics and mutation status of patients with lung adenocarcinoma stratified by PD-L1 and IDO1 expression on tumor cells

\begin{tabular}{|c|c|c|c|c|c|c|c|c|c|}
\hline Variable & PD-L1+ & PD-L1- & $P 1$ & IDO1+ & IDO1- & $P 2$ & IDO1+/PD-L1- & IDO1+/PD-L1+ & $P 3$ \\
\hline$N^{\mathrm{a}}$ & 89 & 172 & & 74 & 186 & & 25 & 49 & \\
\hline Mean age (SD) & $68(10)$ & $68(10)$ & 0.630 & $67(10)$ & $69(10)$ & 0.170 & $64(10)$ & $68(10)$ & 0.380 \\
\hline Sex, $n(\%)$ & & & 0.784 & & & 0.148 & & & 0.383 \\
\hline $\mathrm{F}$ & $57(64)$ & $114(66)$ & & $43(58)$ & $127(68)$ & & $13(52)$ & $30(61)$ & \\
\hline M & $32(36)$ & $58(34)$ & & $31(42)$ & $59(33)$ & & $12(48)$ & $19(39)$ & \\
\hline \multicolumn{10}{|l|}{ Smoking status, $n(\%)$} \\
\hline$\geq 10$ PPY & $81(92)$ & $123(72)$ & $<0.001$ & $63(86)$ & $141(76)$ & 0.089 & $19(76)$ & $44(90)$ & 0.082 \\
\hline$\geq 30$ PPY & $65(75)$ & $84(49)$ & $<0.001$ & $51(70)$ & $98(54)$ & 0.018 & $14(56)$ & $37(76)$ & 0.105 \\
\hline Tumor size, cm (IQR) & $2.1(2)$ & $1.9(2)$ & 0.092 & $2.0(1)$ & $2.0(2)$ & 0.169 & $1.5(1)$ & $2.0(2)$ & 0.070 \\
\hline $\mathrm{T}$ status (pT), $n(\%)$ & & & 0.037 & & & 0.252 & & & 0.011 \\
\hline $\mathrm{T} 1$ & $51(57)$ & $116(67)$ & & $53(72)$ & $113(61)$ & & $20(80)$ & $33(67)$ & \\
\hline $\mathrm{T} 2$ & $33(37)$ & $38(22)$ & & $18(34)$ & $53(29)$ & & $3(12)$ & $15(31)$ & \\
\hline $\mathrm{T} 3$ & $5(6)$ & $13(8)$ & & $3(4)$ & $15(8)$ & & $2(8)$ & $1(2)$ & \\
\hline $\mathrm{T} 4$ & 0 & $5(3)$ & & 0 & $5(3)$ & & 0 & 0 & \\
\hline $\mathrm{N}$ status $(\mathrm{pN})^{\mathrm{b}}, n(\%)$ & & & $\mathbf{0 . 0 3 9}$ & & & 0.310 & & & 1.000 \\
\hline No & $65(73)$ & $147(86)$ & & $59(80)$ & $152(82)$ & & $20(80)$ & $39(80)$ & \\
\hline $\mathrm{N} 1$ & $11(12)$ & $8(5)$ & & $8(11)$ & $11(6)$ & & $2(8)$ & $6(12)$ & \\
\hline $\mathrm{N} 2$ & $8(9)$ & $7(4)$ & & $5(7)$ & $10(5)$ & & $2(8)$ & $3(6)$ & \\
\hline Pathologic stage (AJCC), $n(\%)$ & & & 0.438 & & & 0.917 & & & 1.000 \\
\hline I & $66(74)$ & $135(79)$ & & $59(80)$ & $141(76)$ & & $20(80)$ & $40(82)$ & \\
\hline II & $11(12)$ & $23(13)$ & & $8(11)$ & $26(14)$ & & $2(8)$ & $5(10)$ & \\
\hline III & $11(12)$ & $11(6)$ & & $6(8)$ & $16(9)$ & & $2(8)$ & $4(8)$ & \\
\hline IV & $1(1)$ & $3(2)$ & & $1(1)$ & $3(2)$ & & $1(4)$ & 0 & \\
\hline Predominant pattern (IASLC), $n(\%)$ & & & $<0.001$ & & & $<0.001$ & & & $<0.001$ \\
\hline MIA & $2(2)$ & $18(11)$ & & $3(4)$ & $17(9)$ & & $1(4)$ & $2(4)$ & \\
\hline Lepidic & $8(9)$ & $23(13)$ & & $7(10)$ & $24(13)$ & & $2(8)$ & $5(10)$ & \\
\hline Acinar & $39(44)$ & $65(38)$ & & $40(54)$ & $63(34)$ & & $17(68)$ & $23(47)$ & \\
\hline Papillary & $5(6)$ & $34(20)$ & & $3(4)$ & $36(19)$ & & $2(8)$ & $1(2)$ & \\
\hline Micropapillary & $1(1)$ & $2(1)$ & & $1(1)$ & $2(1)$ & & $1(4)$ & 0 & \\
\hline Solid & $34(38)$ & $9(5)$ & & $18(24)$ & $25(13)$ & & 0 & $18(37)$ & \\
\hline IMA & 0 & $18(11)$ & & $1(1)$ & $17(9)$ & & $1(4)$ & 0 & \\
\hline Fetal & 0 & $3(2)$ & & $1(1)$ & $2(1)$ & & $1(4)$ & 0 & \\
\hline Nuclear grade, $n(\%)$ & & & $<0.001$ & & & 0.007 & & & $<0.001$ \\
\hline 1 & 0 & $7(4)$ & & 0 & $7(4)$ & & 0 & 0 & \\
\hline 2 & $34(38)$ & $131(76)$ & & $39(53)$ & $126(68)$ & & $21(84)$ & $18(37)$ & \\
\hline 3 & $55(62)$ & $34(20)$ & & $35(47)$ & $53(29)$ & & $4(16)$ & $31(63)$ & \\
\hline Pleural invasion, $n(\%)$ & & & 0.207 & & & 0.490 & & & 0.533 \\
\hline 0 & $71(80)$ & $149(87)$ & & $60(81)$ & $159(86)$ & & $19(76)$ & $41(84)$ & \\
\hline 1 & $18(20)$ & $23(13)$ & & $14(19)$ & $27(15)$ & & $6(24)$ & $8(16)$ & \\
\hline Lymphatic invasion, $n(\%)$ & & & $<0.001$ & & & 0.011 & & & 0.051 \\
\hline 0 & $35(39)$ & $122(71)$ & & $35(47)$ & $121(65)$ & & $16(64)$ & $19(39)$ & \\
\hline 1 & $54(61)$ & $50(29)$ & & $39(53)$ & $65(35)$ & & $9(36)$ & $30(61)$ & \\
\hline Vascular invasion, $n(\%)$ & & & $<0.001$ & & & 0.040 & & & 0.290 \\
\hline 0 & $57(64)$ & $152(88)$ & & $53(72)$ & $155(83)$ & & $20(80)$ & $33(67)$ & \\
\hline 1 & $32(36)$ & $20(12)$ & & $21(28)$ & $31(17)$ & & $5(20)$ & $16(33)$ & \\
\hline CD8+ tumor-infiltrating lymphocytes, $n(\%)$ & & & $<0.001$ & & & $<0.001$ & & & $<0.001$ \\
\hline $0-1$ & $30(34)$ & $166(97)$ & & $39(53)$ & $156(84)$ & & $25(100)$ & $14(29)$ & \\
\hline $2-3$ & $59(66)$ & $6(4)$ & & $35(47)$ & $30(16)$ & & 0 & $35(71)$ & \\
\hline CD8+ stroma, $n(\%)$ & & & $<0.001$ & & & $<0.001$ & & & 0.001 \\
\hline$\leq 10 \%$ & $32(36)$ & $133(77)$ & & $33(45)$ & $132(71)$ & & $18(72)$ & $15(31)$ & \\
\hline$>10 \%$ & $57(64)$ & $39(23)$ & & $41(55)$ & $54(29)$ & & $7(28)$ & $34(69)$ & \\
\hline T-bet + tumor-infiltrating lymphocytes, $n(\%)$ & & & $<0.001$ & & & 0.010 & & & 0.073 \\
\hline $0-1$ & $65(73)$ & $166(97)$ & & $59(80)$ & $171(92)$ & & $23(92)$ & $36(74)$ & \\
\hline $2-3$ & $24(27)$ & $6(4)$ & & $15(20)$ & $15(8)$ & & $2(8)$ & $13(27)$ & \\
\hline T-bet+ stroma, $n(\%)$ & & & 0.055 & & & 0.228 & & & 1.000 \\
\hline
\end{tabular}


Table 1 (continued)

\begin{tabular}{|c|c|c|c|c|c|c|c|c|c|}
\hline Variable & PD-L1+ & PD-L1- & $P 1$ & IDO1+ & IDO1- & $P 2$ & IDO1+/PD-L1- & IDO1+/PD-L1+ & $P 3$ \\
\hline$\leq 5 \%$ & $68(76)$ & $149(87)$ & & $58(78)$ & $159(86)$ & & $20(80)$ & $38(78)$ & \\
\hline$>5 \%$ & $21(24)$ & $23(13)$ & & $16(22)$ & $27(15)$ & & $5(20)$ & $11(22)$ & \\
\hline GATA3 + tumor-infiltrating lymphocytes, $n(\%)$ & & & 1.000 & & & 1.000 & & & 1.000 \\
\hline $0-1$ & $88(99)$ & $170(99)$ & & 73 (99) & $184(99)$ & & $25(100)$ & $48(98)$ & \\
\hline $2^{\mathrm{c}}$ & $1(1)$ & $2(1)$ & & $1(1)$ & $2(1)$ & & 0 & $1(2)$ & \\
\hline GATA3+ stroma, $n(\%)$ & & & 0.105 & & & 0.149 & & & 1.000 \\
\hline$\leq 5 \%$ & $70(79)$ & $150(87)$ & & $58(78)$ & $161(87)$ & & $20(80)$ & $38(78)$ & \\
\hline$>5 \%$ & $19(21)$ & $22(13)$ & & $16(22)$ & $25(13)$ & & $5(20)$ & $11(22)$ & \\
\hline Molecular alterations, $n(\%)$ & & & $<0.001$ & & & 0.832 & & & 0.018 \\
\hline KRAS mutations & $46(52)$ & $62(36)$ & & $29(39)$ & $78(42)$ & & $7(28)$ & $22(45)$ & \\
\hline EGFR mutations & $5(6)$ & $49(29)$ & & $13(18)$ & $41(22)$ & & $9(36)$ & $4(8)$ & \\
\hline$A L K$ rearrangements & $1(1)$ & $3(2)$ & & $1(1)$ & $3(2)$ & & $1(4)$ & 0 & \\
\hline Other alterations & $4(5)$ & $9(5)$ & & $4(5)$ & $9(5)$ & & $1(4)$ & $3(6)$ & \\
\hline SNaPshot wild type & $33(37)$ & $49(29)$ & & $27(37)$ & $55(30)$ & & $7(28)$ & $20(41)$ & \\
\hline
\end{tabular}

$P D$ - $L 1$ programmed cell death ligand 1, IDO1 indoleamine 2,3-dioxygenase 1, IQR interquartile range, $P P Y$ pack per year, AJCC American Joint Committee on Cancer, IASLC International Association for the Study of Lung Cancer, MIA minimally invasive adenocarcinoma, IMA invasive mucinous adenocarcinoma, stroma lymphocytes in the tumor stroma

${ }^{\mathrm{a} O n e}$ case did not have assessable IDO1 staining

${ }^{\mathrm{b}} \mathrm{pN}$ data were not available for $N=15$ cases

${ }^{\mathrm{c}}$ No case with a score of 3 for GATA3+ TILs was identified in this cohort

Bold values are significant $P$ values

$(p<0.001)$ and most cases $(18 / 25,72 \%)$ had rare-to-few CD8+ lymphocytes in the tumor stroma $(p=0.001)$.

\section{Correlation of PD-L1 and ID01 expression with HLA class I molecules}

In the entire cohort, 62 (24\%), 234 (90\%), and 250 (96\%) had positive membranous expression of $\mathrm{B} 2 \mathrm{M}, \mathrm{HCA} 2$, and $\mathrm{HC} 10$, respectively, and all cases with positive $\mathrm{B} 2 \mathrm{M}$ membranous expression exhibited $\mathrm{HCA} 2$ and $\mathrm{HC} 10$ staining. Given that $\mathrm{B} 2 \mathrm{M}$ is required for surface expression of $\mathrm{HLA}-\mathrm{A} / \mathrm{B} / \mathrm{C}$, the B2M status was considered to represent the overall HLA class I molecule expression. The HLA class I molecule expression was seen in $32(37 \%)$ of 87 cases with PD-L1 expression and $30(18 \%)$ of 166 cases without; thus, PD-L1 expression was significantly associated with the overall HLA class I molecule expression $(p=0.002)$. Similarly, it was seen in $24(33 \%)$ of 73 IDO1+ and 38 (21\%) of 180 IDO1- cases, and IDO1 expression was marginally associated with overall HLA class I expression $(p=0.054)$. After stratifying the IDO1+ group by PD-L1 expression, there was no significant association between isolated IDO1 expression and overall HLA class I expression $(p=0.300)$.

\section{Correlation of PD-L1 and ID01 expression with molecular alterations}

Molecular alterations were identified in 179 (69\%) of the cohort and included $108(41 \%)$ KRAS mutations, 54 (21\%) EGFR mutations, 4 (2\%) ALK rearrangements, and 13 (5\%) alterations other than $K R A S, E G F R$, and $A L K$. PD-L1 expression was present in 46 (43\%) of $K R A S$ mutants and was significantly associated with KRAS mutations $(p=$ 0.021). Conversely, PD-L1 was positive in only 5 (9\%) of $E G F R$ mutants and was inversely associated with EGFR mutations $(p<0.001)$. In contrast, IDO1 expression was not significantly associated with any molecular alterations. Of the four cases with $A L K$ rearrangements, one expressed PDL1 (weak-to-moderate expression in $100 \%$ of tumor cells) and another expressed IDO1 (weak-to-moderate expression in $40 \%$ of tumor cells). Among the SNaPshot wild-type group, 33/82 (40\%) showed PD-L1 expression and 27/82 (33\%) showed IDO1 expression. There were no differences in the molecular mutational profile between isolated PD-L1 expression and PD-L1/IDO1 co-expression. While there was no association of overall IDO1 expression with molecular alterations, isolated IDO1 expression was seen in 9/13 (69\%) of EGFR mutants and was significantly associated with $E G F R$ mutations $(p=0.007)$.

\section{Logistic regression analysis for PD-L1 and ID01 expression}

Logistic regression analysis revealed that high-grade nuclei (grade 3 vs. grades $1-2, p=0.005$ ), abundant CD8+ tumorinfiltrating lymphocytes $(p<0.001)$, and abundant CD8+ lymphocytes in the tumor stroma $(p=0.032)$ were independent predictors of PD-L1 expression (Table 2). However, only abundant CD8+ tumor-infiltrating lymphocytes independently predicted isolated PD-L1 (PD-L1+/IDO1-) 
Table 2 Logistic regression analysis for correlation of clinicopathologic characteristics and molecular alterations with PDL1 expression

\begin{tabular}{llll}
\hline Predictors of PD-L1 expression & OR & $95 \%$ CI & $P$ \\
\hline Male & 0.54 & $0.20-1.34$ & 0.196 \\
Age & 1.00 & $0.95-1.05$ & 0.987 \\
Smoking ( $\geq 10$ PPY) & 1.33 & $0.39-5.12$ & 0.664 \\
T stage (pT2-4 vs. pT1) & 0.93 & $0.34-2.46$ & 0.888 \\
Nodal stage (pN1-2 vs. pN0) & 2.19 & $0.67-7.19$ & 0.192 \\
Histologic pattern (solid vs. non- & 2.02 & $0.60-6.96$ & 0.254 \\
solid) & & & \\
Nuclear grade (3 vs. 1-2) & 3.80 & $1.50-9.81$ & $\mathbf{0 . 0 0 5}$ \\
Lymphatic invasion (present vs. & 1.93 & $0.65-5.69$ & 0.230 \\
absent) & & & \\
Vascular invasion (present vs. & 1.56 & $0.50-4.87$ & 0.437 \\
absent) & & & \\
CD8+ tumor-infiltrating & 29.53 & $9.74-108.02$ & $<\mathbf{0 . 0 0 1}$ \\
lymphocytes (2-3 vs. 0-1) & & & \\
CD8+ stroma (>10\% vs. $\leq 10 \%)$ & 2.65 & $1.08-6.51$ & $\mathbf{0 . 0 3 2}$ \\
T-bet+ tumor-infiltrating & 0.70 & $0.13-3.99$ & 0.676 \\
lymphocytes (2-3 vs. 0-1) & & & \\
KRAS mutations & 1.99 & $0.75-5.57$ & 0.177 \\
$E G F R$ mutations & 0.29 & $0.06-1.21$ & 0.107 \\
\hline$P D-L 1$ programmed cell death & & & \\
\hline
\end{tabular}

$P D-L 1$ programmed cell death ligand 1, $P P Y$ pack per year, $O R$ odds ratio, $C I$ confidence interval, stroma lymphocytes in the tumor stroma Bold values are significant $P$ values

expression (odds ratio $(\mathrm{OR})=4.20,95 \%$ confidence interval (CI) $1.40-13.02, \quad p=0.011)$. For IDO1, logistic regression analysis revealed that younger age $(p=0.033)$, acinar-predominant histological pattern $(p=0.005)$, and abundant CD8 + tumor-infiltrating lymphocytes $(p=0.009)$ were independent predictors of expression (Table 3). However, the association with abundant CD8+ tumorinfiltrating lymphocytes disappeared with isolated IDO1 (IDO1+/PD-L1-) expression, while the association with acinar-predominant histologic pattern remained significant $(\mathrm{OR}=5.31,95 \%$ CI $1.84-16.91, p=0.003)$. The analyses were adjusted for sex, age, and factors that were significantly associated with PD-L1 or IDO1 expression, respectively, using univariate analysis.

\section{Survival analysis}

Patients with lung adenocarcinomas expressing PD-L1 had significantly lower 5-year progression-free survival $(65 \%$ vs. $75 \%, \log \operatorname{rank} p=0.005)$ and overall survival (74\% vs. $83 \%, \log$ rank $p=0.011)$ compared to patients whose tumors did not express PD-L1 (Fig. 3). However, by multivariate analysis, PD-L1 was no longer significantly associated with progression-free and overall survival. In the multivariate model, higher T stage (pT2-4 vs. pT1) was
Table 3 Logistic regression analysis for correlation of clinicopathologic characteristics and molecular alterations with IDO1 expression

\begin{tabular}{llll}
\hline & OR & $95 \%$ CI & $P$ \\
\hline Male & 1.65 & $0.87-3.13$ & 0.126 \\
Age & 0.97 & $0.94-1.00$ & $\mathbf{0 . 0 3 3}$ \\
Smoking ( $\geq 30$ PPY) & 1.58 & $0.82-3.07$ & 0.172 \\
Histologic pattern (acinar vs. non-acinar) & 2.42 & $1.31-4.53$ & $\mathbf{0 . 0 0 5}$ \\
Nuclear grade (3 vs. 1-2) & 1.12 & $0.56-2.20$ & 0.746 \\
Lymphatic invasion (present vs. absent) & 1.01 & $0.49-2.03$ & 0.976 \\
Vascular invasion (present vs. absent) & 1.36 & $0.62-3.00$ & 0.441 \\
CD8+ tumor-infiltrating lymphocytes $(2-3$ & 2.94 & $1.31-6.64$ & $\mathbf{0 . 0 0 9}$ \\
vs. 0-1) & & & \\
CD8+ stroma (>10\% vs. $\leq 10 \%)$ & 1.71 & $0.87-3.34$ & 0.118 \\
T-bet+ tumor-infiltrating lymphocytes & 1.26 & $0.48-3.27$ & 0.638 \\
(2-3 vs. 0-1) & & & \\
\hline
\end{tabular}

IDO1 indoleamine 2,3-dioxygenase 1, $P P Y$ pack per year, $O R$ odds ratio, $C I$ confidence interval, stroma lymphocytes in the tumor stroma Bold values are significant $P$ values

associated with shorter progression-free and overall survival; histologic pattern (solid vs. non-solid) and lymphatic and vascular invasion were associated with shorter progression-free survival. There was no significant relationship between CD8+ tumor-infiltrating lymphocytes or $\mathrm{CD} 8+$ lymphocytes in the stroma with progression-free or overall survival (Supplementary Table 2).

On the contrary, patients with lung adenocarcinomas expressing IDO1 did not show a significant difference in 5year progression-free survival $(67 \%$ vs. $73 \%, \log$ rank $p=$ $0.147)$ or overall survival $(77 \%$ vs. $81 \%, \log$ rank $p=$ 0.199) compared to those with negative IDO1 expression (Fig. 4). Similarly, multivariate analysis did not reveal a significant association between IDO1 and progression-free or overall survival. In this model, lymphatic and vascular invasion were associated with shorter progression-free survival, and significant smoking history was associated with shorter overall survival. As with PD-L1, there was no significant relationship between CD8+ tumor-infiltrating lymphocytes or CD8+ lymphocytes in the stroma with progression-free or overall survival (Supplementary Table 3).

Interestingly, stratification into the four expression groups (PD-L1-/IDO1-, PD-L1+/IDO1-, PD-L1-/IDO1 + , and PD-L1+/IDO1+) revealed a significant difference in 5 -year progression-free survival $(p=0.004)$ and overall survival $(p=0.038)$ among the four groups (Fig. 5). The 5year progression-free survival rates were $77 \%, 55 \%, 62 \%$, and $71 \%$ for PD-L1-/IDO1-, PD-L1+/IDO1-, PD-L1 $-/ \mathrm{IDO} 1+$, and PD-L1+/IDO1+ tumors, respectively. The 5 -year overall survival rates were $84 \%, 68 \%, 77 \%$, and $78 \%$ for PD-L1-/IDO1-, PD-L1+/IDO1-, PD-L1-/IDO1+, 

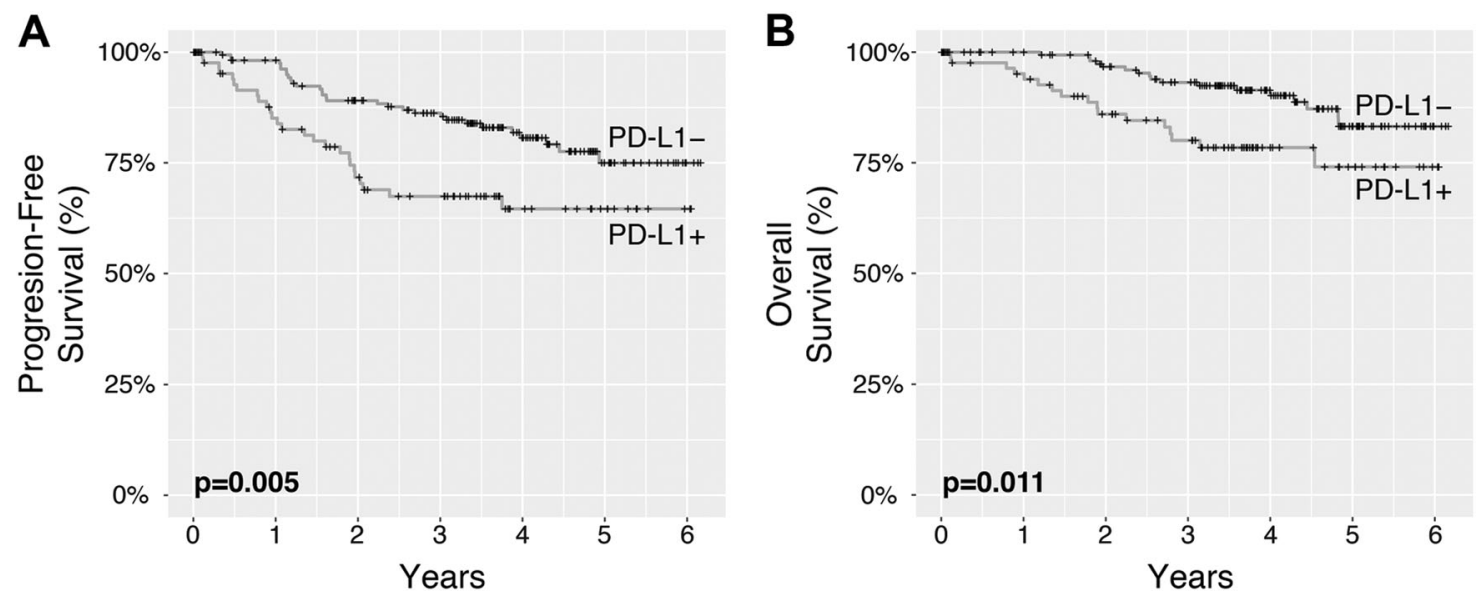

Fig. 3 Kaplan-Meier curves showing (a) progression-free survival and (b) overall survival of lung adenocarcinoma patients with positive and negative PD-L1 expression (cutoff of $\mathrm{H}$-score $=5$ )
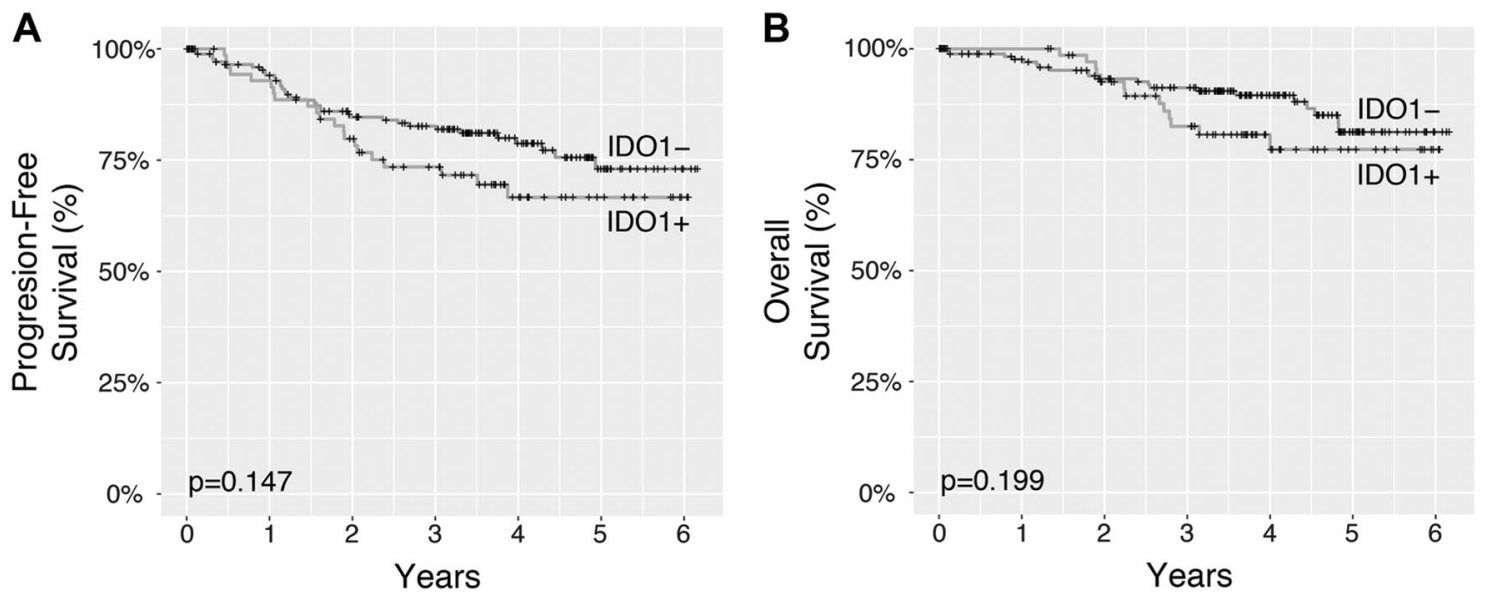

Fig. 4 Kaplan-Meier curves showing (a) progression-free survival and (b) overall survival of lung adenocarcinoma patients with positive and negative IDO1 expression (cutoff of $\mathrm{H}$-score $=5$ )

and PD-L1+/IDO1+ tumors, respectively. Tumors that were negative for both PD-L1 and IDO1 had the best survival, while those with isolated PD-L1 expression had the worst survival; expression of IDO1 conferred an intermediate survival.

\section{Discussion}

In this study, we confirm that PD-L1 expression (with or without IDO1 co-expression) is observed in association with $\mathrm{CD} 8+$ and T-bet + tumor-infiltrating lymphocytes, HLA class I expression, and KRAS mutations $[9,30]$. We also corroborate the inverse association of PD-L1 with $E G F R$ mutations, which is consistent with data suggesting that the EGFR-mutant status confers diminished response to PD-1 blockade $[2,31]$. This, together with the association between PD-L1 and the cytotoxic T lymphocyte/Th1 microenvironment, supports the role of PD-L1 upregulation in adaptive immune resistance as opposed to innate immune resistance, the latter of which may be seen in EGFR-mutant and $A L K$-rearranged non-small cell lung cancer [32, 33]. In contrast to PD-L1, isolated IDO1 expression was significantly associated with the absence of CD8+ tumorinfiltrating lymphocytes along with a lower pT stage and lower nuclear grade. Furthermore, significantly more cases with EGFR mutations had IDO1 expression without coexpression of PD-L1.

To date, the biological role of additional potentially actionable immune inhibitory targets and their associations with PD-L1 are extremely limited in lung cancer. Clinical trials of IDO1 inhibitors as a single agent (NCT01219348 [19], NCT03164603, NCT03208959) and in combination with pembrolizumab/nivolumab (NCT02752074, 
A

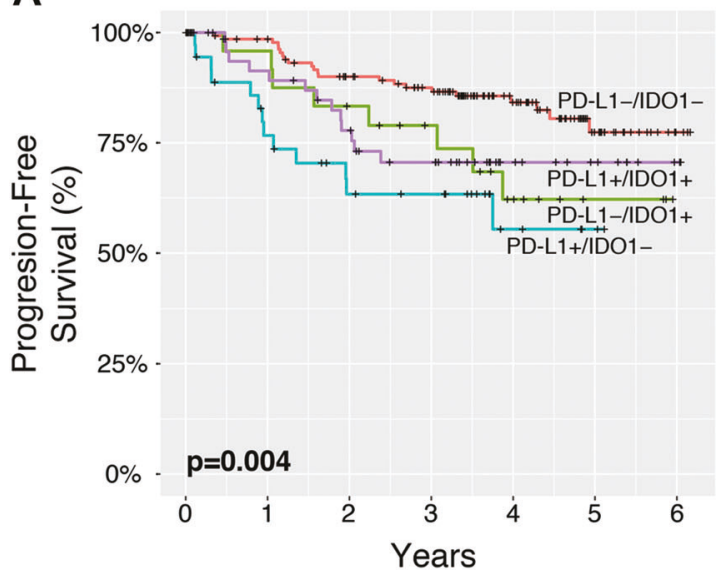

B

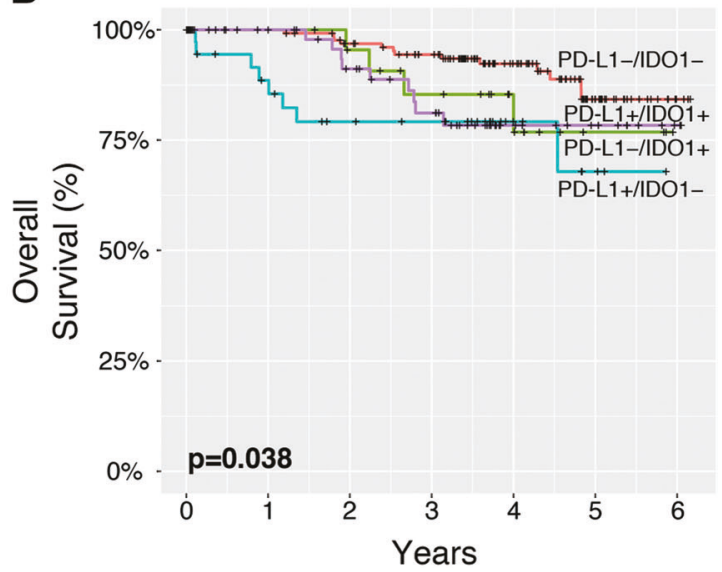

Fig. 5 Kaplan-Meier curves showing a progression-free survival and b overall survival of lung adenocarcinoma patients with PD-L1-/IDO1-, PD-L1+/IDO1-, PD-L1-/IDO1+, and PD-L1+/IDO1+ expression (cutoff of H-score =5)

NCT03301636, NCT02658890, NCT02178722) for patients with non-small cell lung cancer are currently underway. However, the only evidence for the biopathological role of IDO1 in lung cancer are that IDO1 promotes tumorigenesis in mouse models of primary KRASinduced lung adenocarcinoma [15] and that out of carcinomas, human non-small cell lung cancer is the third most frequent expresser of IDO1 after endometrial/cervical carcinomas and renal carcinomas [13]. In our cohort, 49/260 (19\%) cases co-expressed PD-L1 and IDO1, while 40 (15\%) and 25 (10\%) cases expressed only PD-L1 and only IDO1, respectively. Interestingly, recent studies have reported that $7-29 \%$ of tumors co-express PD-L1 and IDO1 in 4 non-small cell lung cancer cohorts (Table 4) [21, 34, 35]. The discrepant results could be attributed to the differences in the study cohorts (adenocarcinoma only vs. all types of non-small cell lung cancer, ethnicity), the detection assays used (immunohistochemistry vs. immunofluorescence), and the cutoff values. It is reasonable to see some extent of PD-L1 and IDO1 co-expression in tumor cells, however, given that the IFN $\gamma$ can induce expression of those immune inhibitory proteins $[21,36]$.

In the current study, overall IDO1 expression was significantly associated with a heavy smoking history $(\geq 30$ pack-years, but not $\geq 10$ ), more aggressive pathological factors, and abundant CD8+/T-bet + tumor-infiltrating lymphocytes. A recent study of a smaller non-small cell lung cancer cohort also showed that upregulation of IDO1 expression was significantly correlated with a higher pathologic stage as well as lymph node metastasis (Table 4) [37], which was not seen in the present study. There was no association between overall IDO1 expression and molecular alterations. Younger age and abundant CD8+ tumorinfiltrating lymphocytes were independent predictors of overall IDO1 expression by multivariate analysis. At first glance, these initial analyses suggest close similarities between the relationships of PD-L1 and IDO1 expression with clinicopathologic factors; however, further stratification into isolated IDO1 expression versus IDO1 with PD-L1 co-expression elucidated notable differences. Isolated IDO1 expression did not show an association with significant smoking history. Interestingly, some previous associations with overall IDO1 expression changed with isolated IDO1 expression: IDO1 expression alone was significantly associated with less aggressive pathologic factors (i.e., lower nuclear grade) and rare/few CD8+ tumor-infiltrating lymphocytes/stromal lymphocytes, and was no longer associated with T-bet+ tumor-infiltrating lymphocytes. Abundant CD8+ tumor-infiltrating lymphocytes were not seen in any cases with isolated IDO1 expression. These findings suggest that a mechanism(s) distinct from the cytotoxic T lymphocyte/Th1 microenvironment may facilitate the isolated IDO1 expression. For instance, activation of RAS and PAMP (pathogen-associated molecular pattern) along with IFN $\gamma / \mathrm{JAK} 2 / \mathrm{STAT}$ (interferon- $\gamma / \mathrm{Janus}$ kinase 2/ signal transducer and activator of transcription) pathways in tumor cells can induce IDO1 [36]. Of those, the latter is shared with PD-L1 expression. In the current cohort, the isolated IDO1 expression was associated with EGFR mutations that activate RAS signaling and often exhibit lower nuclear grade and limited inflammatory infiltrate [3840]. The association raises the possibility of treatment with IDO1 inhibitors for EGFR-mutated non-small cell lung cancer, for which PD-1/PD-L1 blockade is generally not effective. These findings, however, need to be confirmed by additional studies with larger IDO1+ cohorts.

PD-L1 expression was significantly associated with expression of HLA class I molecules, while abundant CD8+ tumor-infiltrating lymphocytes were also associated with HLA class I expression (data not shown). This is consistent 


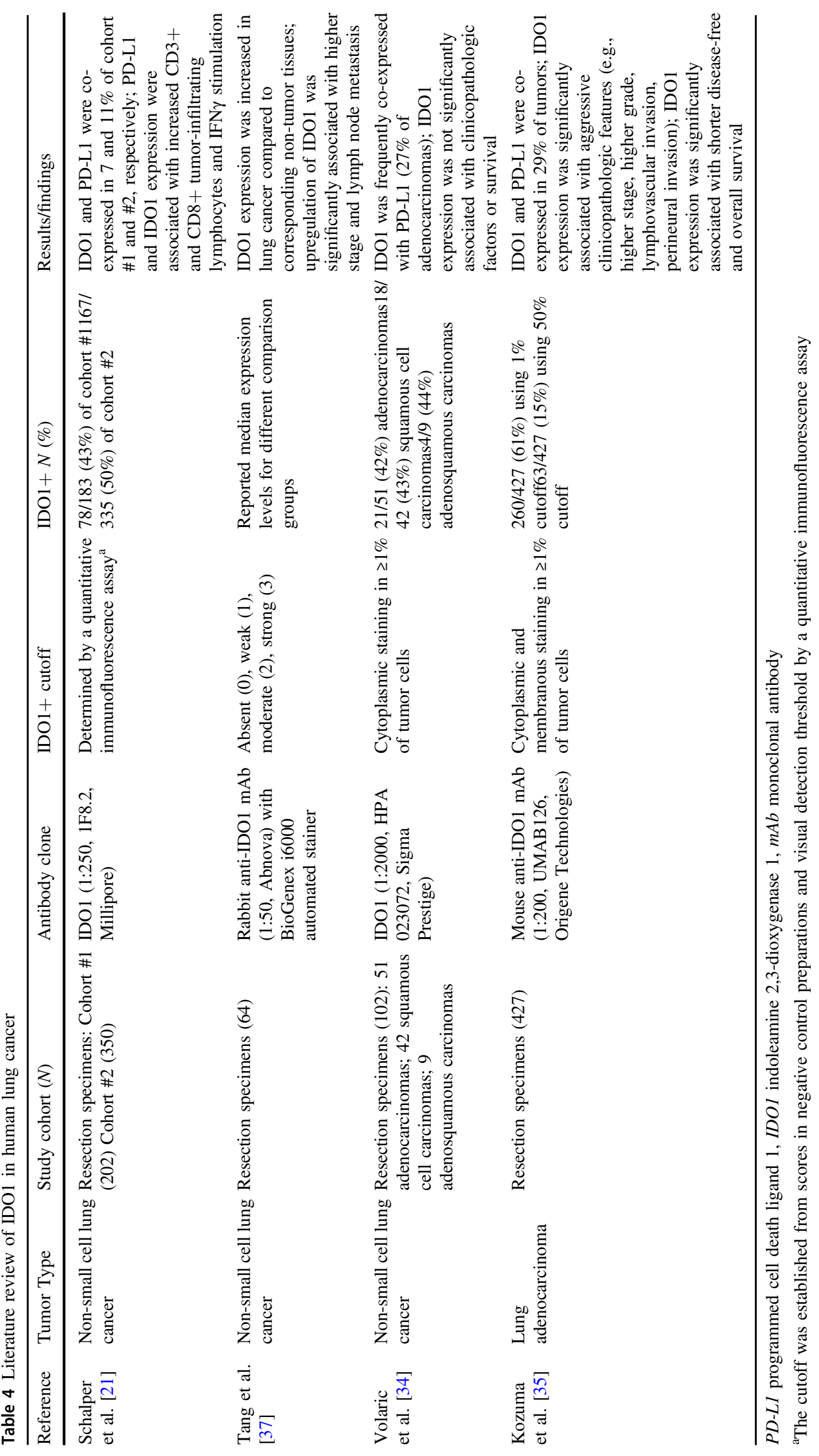


with the mechanism of adaptive immune resistance, whereby antigen presentation via HLA class I molecules on the surface of tumor cells recruits CD8+ cytotoxic T lymphocytes that subsequently produce IFN $\gamma$ and drive upregulation of PD-L1 in tumor cells, leading to the deactivation of $\mathrm{CD} 8+$ tumor-infiltrating lymphocytes via PD-L1/PD-1 interactions. Thus, it follows that downregulation of HLA class I expression likely confers a worse prognosis in cancers, including non-small cell lung cancer [41, 42]. In contrast, neither overall IDO1 nor isolated IDO1 expression were associated with HLA class I molecule expression in this study, supporting a different mechanism of immune suppression within the tumor microenvironment, possibly involving other types of $\mathrm{T}$ lymphocytes such as regulatory $\mathrm{T}$ cells, B cells and/or myeloid-derived suppressor cells [16, $21,36]$.

Regarding patient outcomes, PD-L1 expression was associated with decreased progression-free and overall survival by univariate but not multivariate analysis, while multiple studies have reported conflicting results regarding the prognostic utility of PD-L1 expression, including correlations with poorer [10, 43, 44], improved [11, 45, 46], and no difference $[12,21]$ in overall survival. In this study, there was no difference in progression-free or overall survival between tumors expressing IDO1 and those without IDO1 expression, confirming the results of the non-small cell lung cancer study by Volaric et al. [34]. In contrast, a few other studies involving lung adenocarcinoma and other cancer types have reported a correlation between high IDO1 expression in tumor cells and decreased survival [35, 47, 48]. Thus, additional large-scale studies are warranted to determine the prognostic role of IDO1 in non-small cell lung cancer. Interestingly, however, after separating expression of PD-L1 and IDO1 in these cases, PD-L1 +/IDO1 - tumors showed the shortest progression-free and overall survival, whereas PD-L1/IDO1 double-negative tumors showed the longest progression-free and overall survival and tumors expressing IDO1 had an intermediate survival outcome. These findings raise the possibility that while IDO1 expression does contribute to immune resistance and tumor progression, it acts via mechanisms separate and not necessarily synergistic with that of PD-L1, thus conferring a slight survival advantage over tumors expressing PD-L1 but not IDO1.

Our study has several important limitations. First, there is currently no gold standard in PD-L1 or IDO1 testing, and there exist a number of different antibodies and scoring systems $[49,50]$. To account for this, we used a commercially available anti-PD-L1 antibody that has been independently validated $[10,12,51]$ and an IDO1 antibody that was validated in a previous study using non-small cell lung cancer tissue microarrays [21]. Second, we chose to use Hscoring and a cutoff of $\mathrm{H}$-score $\geq 5$ as the definition of positive expression for both PD-L1 and IDO1 immunohistochemistry to account for both the extent and intensity of expression. However, there is heterogeneity of the scoring systems and cutoffs for positive expression used across different studies, making it difficult for direct comparison. Previously on this cohort, Huynh et al. [9]. analyzed PD-L1 expression using $\mathrm{H}$-score (cutoff $\geq 5$ ), immune score ( 3 vs. $0-2$ ), and extent of expression of any intensity (cutoffs of $1 \%, 5 \%$, and $50 \%$ ), and achieved similar results across the different scoring systems. Using an $\mathrm{H}$-score cutoff of $\geq 5$ performed most similarly to an expression cutoff of $\geq 5 \%$. Another issue is the use of tissue microarrays, which only represent small portions of the tumor and thus makes it difficult to evaluate for heterogeneous expression within a tumor, as recently reported [52-55]. To overcome the issue of heterogeneity, we constructed tissue microarrays of two larger-caliber cores from each of the cohort cases, where one core was taken from the tumor center and the other from the invasive front. The final $\mathrm{H}$-score was determined based on their collective assessment. Of note, the two cores had discrepant scores in 7\% (PD-L1) and 5\% (IDO1) of our study cases (data not shown), and the discrepancy rate of PD-L1 was smaller than those observed between biopsies or tissue microarray cores and whole tumor sections [52, 53]. Furthermore, corresponding whole sections from a subset of the study cohort (28 cases, $11 \%$ ) with a range of IDO1 expression on tissue microarray (including the vast majority of those with discrepant cores) were evaluated for IDO1 expression and showed strong, significant correlation in $\mathrm{H}-$ score with the tissue microarray cores (Pearson's $r=0.7, p$ $<0.001)$. Using an $\mathrm{H}$-score cutoff of $\geq 5$, only one case $(4 \%)$ showed discrepant IDO1 expression $(\mathrm{H}$-score $=4$ on tissue microarray and 8 on the corresponding whole section). Finally, our study cohort consisted of mostly white, female patients with early-stage tumors (reflective of the resected primary lung adenocarcinoma population at our institution) and limited molecular data regarding $A L K$ rearrangementpositive cases (only $n=4$ ) and ROS1 rearrangementpositive cases ( $n=65$ evaluated by ROSI fluorescence in situ hybridization, but none positive).

Further large-scale studies are needed to elucidate the roles and interactions of PD-L1 with IDO1 and other immunosuppressive markers. Additional questions include the expression of IDO1 after anti-PD-1/PD-L1 blockade [56] and whether it may play a role in immunotherapy resistance, the relationship between IDO1 and HLA class II markers, and the role of PD-L1 and/or IDO1 expression in rearrangement-positive non-small cell lung cancer.

Overall, our results confirm that PD-L1 expression with or without IDO1 co-expression is associated with the cytotoxic $\mathrm{T}$ lymphocyte/Th1 immune microenvironment. IDO1 appears to serve an immunosuppressive role distinct from and possibly not synergistic to that of PD-L1, 
supporting the notion that lung adenocarcinomas use a combination of mechanisms to evade tumor immunity. As there remains a need for biomarkers to identify tumors that may be resistant to PD-1/PD-L1 blockade, further investigation of IDO1 as a possible biomarker may be warranted.

Funding This work was supported by a Stand Up To CancerAmerican Cancer Society Dream Team Translation Research Grant (to JFG and MM-K).

\section{Compliance with ethical standards}

Conflict of interest AJI has equity interest and royalties from exclusive license of AMP technology to ArcherDx and served as a scientific advisory board member for ArcherDx. JFG served as a compensated consultant or received honoraria from Bristol-Myers Squibb, Merck, Genentech/Roche, Ariad, Takeda, Pfizer, Amgen, Agios, Array Biopharma, Novartis, Incyte, and Loxo. MM-K served as a compensated consultant for Merrimack Pharmaceuticals and H3 Biomedicine. The other authors declare that they have no conflict of interest.

\section{References}

1. Pardoll DM. The blockade of immune checkpoints in cancer immunotherapy. Nat Rev Cancer. 2012;12:252-64.

2. Borghaei H, Paz-Ares L, Horn L, et al. Nivolumab versus docetaxel in advanced nonsquamous non-small-cell lung cancer. $\mathrm{N}$ Engl J Med. 2015;373:1627-39.

3. Reck M, Rodríguez-Abreu D, Robinson AG, et al. Pembrolizumab versus chemotherapy for PD-L1-positive non-smallcell lung cancer. N Engl J Med. 2016;375:1823-33.

4. Fehrenbacher L, Spira A, Ballinger M, et al. Atezolizumab versus docetaxel for patients with previously treated non-small-cell lung cancer (POPLAR): a multicentre, open-label, phase 2 randomised controlled trial. Lancet. 2016;387:1837-46.

5. Rittmeyer A, Barlesi F, Waterkamp D, et al. Atezolizumab versus docetaxel in patients with previously treated non-small-cell lung cancer (OAK): a phase 3, open-label, multicentre randomised controlled trial. Lancet. 2017;389:255-65.

6. Brahmer J, Reckamp KL, Baas P, et al. Nivolumab versus docetaxel in advanced squamous-cell non-small-cell lung cancer. $\mathrm{N}$ Engl J Med. 2015;373:123-35.

7. Langer CJ, Gadgeel SM, Borghaei H, et al. Carboplatin and pemetrexed with or without pembrolizumab for advanced, nonsquamous non-small-cell lung cancer: a randomised, phase 2 cohort of the open-label KEYNOTE-021 study. Lancet Oncol. 2016;17:1497-508.

8. Gandhi L, Rodríguez-Abreu D, Gadgeel S, et al. Pembrolizumab plus chemotherapy in metastatic non-small-cell lung cancer. $\mathrm{N}$ Engl J Med. 2018;378:2078-92.

9. Huynh TG, Morales-Oyarvide V, Campo MJ, et al. Programmed cell death ligand 1 expression in resected lung adenocarcinomas: association with immune microenvironment. J Thorac Oncol. 2016;11:1869-78.

10. Shimoji M, Shimizu S, Sato K, et al. Clinical and pathologic features of lung cancer expressing programmed cell death ligand 1 (PD-L1). Lung Cancer. 2016;98:69-75.

11. Cooper WA, Tran T, Vilain RE, et al. PD-L1 expression is a favorable prognostic factor in early stage non-small cell carcinoma. Lung Cancer. 2015;89:181-8.

12. Schmidt LH, Kümmel A, Görlich D. et al. PD-1 and PD-L1 expression in non-small cell lung cancer indicate a favorable prognosis in defined subgroups. PLoS ONE. 2015;10:e0136023
13. Théate I, van Baren N, Pilotte L, et al. Extensive profiling of the expression of the indoleamine 2,3-dioxygenase 1 protein in normal and tumoral human tissues. Cancer Immunol Res. 2015;3:161-72.

14. Xie Q, Wang L, Zhu B, Wang Y, Gu J, Chen Z. [The expression and significance of indoleamine -2,3 -dioxygenase in non-small cell lung cancer cell.]. Zhongguo Fei Ai Za Zhi. 2008;11:115-9.

15. Smith C, Chang MY, Parker KH, et al. IDO is a nodal pathogenic driver of lung cancer and metastasis development. Cancer Discov. 2012;2:722-35.

16. Zhai L, Spranger S, Binder DC, et al. Molecular pathways: targeting IDO1 and other tryptophan dioxygenases for cancer immunotherapy. Clin Cancer Res. 2015;21:5427-33.

17. Ferdinande L, Decaestecker C, Verset L, et al. Clinicopathological significance of indoleamine 2,3-dioxygenase 1 expression in colorectal cancer. Br J Cancer. 2012;106:141-7.

18. Spranger S, Koblish HK, Horton B, Scherle PA, Newton R, Gajewski TF. Mechanism of tumor rejection with doublets of cytotoxic T lymphocyteA-4, PD-1/PD-L1, or IDO blockade involves restored IL-2 production and proliferation of $\mathrm{CD} 8(+)$ $\mathrm{T}$ cells directly within the tumor microenvironment. J Immunother Cancer. 2014;2:3.

19. Iversen TZ, Engell-Noerregaard L, Ellebaek E, et al. Long-lasting disease stabilization in the absence of toxicity in metastatic lung cancer patients vaccinated with an epitope derived from indoleamine 2,3 dioxygenase. Clin Cancer Res. 2014;20:221-32.

20. Moon YW, Hajjar J, Hwu P, Naing A. Targeting the indoleamine 2,3-dioxygenase pathway in cancer. $\mathrm{J}$ Immunother Cancer. 2015;3:51.

21. Schalper KA, Carvajal-Hausdorf D, McLaughlin J, et al. Differential expression and significance of PD-L1, IDO-1, and B7-H4 in human lung cancer. Clin Cancer Res. 2017;23:370-8.

22. Dias-Santagata D, Akhavanfard S, David SS, et al. Rapid targeted mutational analysis of human tumours: a clinical platform to guide personalized cancer medicine. EMBO Mol Med. 2010;2: 146-58.

23. Travis WD, Brambilla E, Noguchi M, et al. International Association for the Study of Lung Cancer/American Thoracic Society/ European Respiratory Society International Multidisciplinary Classification of Lung Adenocarcinoma. $\mathrm{J}$ Thorac Oncol. 2011;6:244-85.

24. Detterbeck FC, Boffa DJ, Tanoue LT. The new lung cancer staging system. Chest. 2009;136:260-71.

25. Barletta JA, Yeap BY, Chirieac LR. Prognostic significance of grading in lung adenocarcinoma. Cancer . 2010;116:659-69.

26. Stam NJ, Vroom TM, Peters PJ, Pastoors EB, Ploegh HL. HLAA- and HLA-B-specific monoclonal antibodies reactive with free heavy chains in western blots, in formalin-fixed, paraffinembedded tissue sections and in cryo-immuno-electron microscopy. Int Immunol. 1990;2:113-25.

27. Ryu KS, Lee YS, Kim BK, et al. Alterations of HLA class I and II antigen expression in preinvasive, invasive and metastatic cervical cancers. Exp Mol Med. 2001;33:136-44.

28. Torigoe T, Asanuma H, Nakazawa E, et al. Establishment of a monoclonal anti-pan HLA class I antibody suitable for immunostaining of formalin-fixed tissue: unusually high frequency of down-regulation in breast cancer tissues. Pathol Int. 2012;62:303-8.

29. Rosenbaum MW, Gigliotti BJ, Pai SI, et al. PD-L1 and IDO1 are expressed in poorly differentiated thyroid carcinoma. Endocr Pathol. 2018;29:59-67.

30. Dacic S, Shuai Y, Yousem S, Ohori P, Nikiforova M. Clinicopathological predictors of EGFR/KRAS mutational status in primary lung adenocarcinomas. Mod Pathol. 2010;23:159-68.

31. Gainor JF, Shaw AT, Sequist LV, et al. EGFR mutations and ALK rearrangements are associated with low response rates to 
PD-1 pathway blockade in non-small cell lung cancer: a retrospective analysis. Clin Cancer Res. 2016;22:4585-93.

32. Akbay EA, Koyama S, Carretero J, et al. Activation of the PD-1 pathway contributes to immune escape in EGFR-driven lung tumors. Cancer Discov. 2013;3:1355-63.

33. Ota K, Azuma K, Kawahara A, et al. Induction of PD-L1 expression by the EML4-ALK oncoprotein and downstream signaling pathways in non-small cell lung cancer. Clin Cancer Res. 2015;21:4014-21.

34. Volaric A, Gentzler R, Hall R, et al. Indoleamine-2,3-dioxygenase in non-small cell lung cancer. Am J Surg Pathol. 2018;42:1216-23.

35. Kozuma Y, Takada K, Toyokawa G, et al. Indoleamine 2,3dioxygenase 1 and programmed cell death-ligand 1 co-expression correlates with aggressive features in lung adenocarcinoma. Eur J Cancer. 2018;101:20-29.

36. Prendergast GC, Malachowski WP, DuHadaway JB, Muller AJ. Discovery of IDO1 inhibitors: from bench to bedside. Cancer Res. 2017;77:6795-811.

37. Tang D, Yue L, Yao R, et al. P53 prevent tumor invasion and metastasis by down-regulating IDO in lung cancer. Oncotarget. 2017;8:54548-57.

38. Lin C-Y, Wu Y-M, Hsieh M-H, et al. Prognostic implication of EGFR gene mutations and histological classification in patients with resected stage I lung adenocarcinoma. PLoS ONE. 2017;12: e0186567.

39. Levy M, Lyon L, Barbero E, et al. Histologic grade is predictive of incidence of epidermal growth factor receptor mutations in metastatic lung adenocarcinoma. Med Sci (Basel, Switz). 2017;5: e34.

40. Sharma S, Gupta N, Singh N, Chaturvedi R, Behera D, Rajwanshi A. Cytomorphological features as predictors of epidermal growth factor receptor mutation status in lung adenocarcinoma. Cytojournal. 2018;15:11.

41. Chang C-C, Campoli M, Ferrone S. HLA class I defects in malignant lesions: what have we learned? Keio J Med. 2003;52:220-9.

42. Hanagiri T, Shigematsu Y, Shinohara S, et al. Clinical significance of expression of cancer/testis antigen and down-regulation of HLA class-I in patients with stage I non-small cell lung cancer. Anticancer Res. 2013;33:2123-8.

43. Zhang Y, Wang L, Li Y, et al. Protein expression of programmed death 1 ligand 1 and ligand 2 independently predict poor prognosis in surgically resected lung adenocarcinoma. Onco Targets Ther. 2014;7:567-73.

44. Azuma K, Ota K, Kawahara A, et al. Association of PD-L1 overexpression with activating EGFR mutations in surgically resected nonsmall-cell lung cancer. Ann Oncol J Eur Soc Med Oncol. 2014;25:1935-40.

45. Velcheti V, Schalper KA, Carvajal DE, et al. Programmed death ligand-1 expression in non-small cell lung cancer. Lab Investig. 2014;94:107-16.

46. D'Incecco A, Andreozzi M, Ludovini V, et al. PD-1 and PD-L1 expression in molecularly selected non-small-cell lung cancer patients. Br J Cancer. 2015;112:95-102.

47. Takao M, Okamoto A, Nikaido T, et al. Increased synthesis of indoleamine-2,3-dioxygenase protein is positively associated with impaired survival in patients with serous-type, but not with other types of, ovarian cancer. Oncol Rep. 2007;17:1333-9.

48. Zhai L, Ladomersky $\mathrm{E}$, Lauing $\mathrm{KL}$, et al. Infiltrating $\mathrm{T}$ cells Increase IDO1 expression in glioblastoma and contribute to decreased patient survival. Clin Cancer Res. 2017;23:6650-60.

49. Mino-Kenudson M. Programmed cell death ligand-1 (PD-L1) expression by immunohistochemistry: could it be predictive and/ or prognostic in non-small cell lung cancer? Cancer Biol Med. 2016;13:157-70.

50. Sholl LM, Aisner DL, Allen TC, et al. Programmed death ligand-1 immunohistochemistry - a new challenge for pathologists: a perspective from members of the Pulmonary Pathology Society. Arch Pathol Lab Med. 2016;140:341-4.

51. Koh J, Go H, Keam B, et al. Clinicopathologic analysis of programmed cell death-1 and programmed cell death-ligand 1 and 2 expressions in pulmonary adenocarcinoma: comparison with histology and driver oncogenic alteration status. Mod Pathol. 2015;28:1154-66.

52. Ilie M, Long-Mira E, Bence C, et al. Comparative study of the PD-L1 status between surgically resected specimens and matched biopsies of non-small cell lung cancer patients reveal major discordances: a potential issue for anti-PD-L1 therapeutic strategies. Ann Oncol J Eur Soc Med Oncol. 2016;27:147-53.

53. McLaughlin J, Han G, Schalper KA, et al. Quantitative assessment of the heterogeneity of PD-L1 expression in non-small-cell lung cancer. JAMA Oncol. 2016;2:46.

54. Rehman JA, Han G, Carvajal-Hausdorf DE, et al. Quantitative and pathologist-read comparison of the heterogeneity of programmed death-ligand 1 (PD-L1) expression in non-small cell lung cancer. Mod Pathol. 2017;30:340-9.

55. Uruga H, Bozkurtlar E, Huynh TG, et al. Programmed cell death ligand (PD-L1) expression in stage II and III lung adenocarcinomas and nodal metastases. J Thorac Oncol. 2017;12:458-66.

56. Li A, Barsoumian HB, Schoenhals JE, et al. Indoleamine 2,3dioxygenase 1 inhibition targets anti-PD1-resistant lung tumors by blocking myeloid-derived suppressor cells. Cancer Lett. 2018;431:54-63. 